\title{
Pascu-Type Harmonic Functions with Positive Coefficients Involving Salagean Operator
}

\author{
K. Vijaya, G. Murugusundaramoorthy, and M. Kasthuri \\ School of Advanced Sciences, VIT University, Vellore 632014, India \\ Correspondence should be addressed to K. Vijaya; kvijaya@vit.ac.in \\ Received 25 November 2013; Accepted 21 February 2014; Published 6 April 2014 \\ Academic Editor: Remi Léandre
}

Copyright ( $2014 \mathrm{~K}$. Vijaya et al. This is an open access article distributed under the Creative Commons Attribution License, which permits unrestricted use, distribution, and reproduction in any medium, provided the original work is properly cited.

Making use of a Salagean operator, we introduce a new class of complex valued harmonic functions which are orientation preserving and univalent in the open unit disc. Among the results presented in this paper including the coeffcient bounds, distortion inequality, and covering property, extreme points, certain inclusion results, convolution properties, and partial sums for this generalized class of functions are discussed.

\section{Introduction and Preliminaries}

A continuous function $f=u+i v$ is a complex-valued harmonic function in a complex domain $\mathscr{G}$ if both $u$ and $v$ are real and harmonic in $\mathscr{G}$. In any simply connected domain $\mathscr{D} \subset \mathscr{G}$, we can write $f=h+\bar{g}$, where $h$ and $g$ are analytic in $\mathscr{D}$. We call $h$ the analytic part and $g$ the coanalytic part of $f$. A necessary and sufficient condition for $f$ to be locally univalent and orientation preserving in $\mathscr{D}$ is that $\left|h^{\prime}(z)\right|>$ $\left|g^{\prime}(z)\right|$ in $\mathscr{D}$ (see [1]).

Denote by $\mathscr{H}$ the family of functions

$$
f=h+\bar{g}
$$

which are harmonic, univalent, and orientation preserving in the open unit disc $\mathcal{U}=\{z:|z|<1\}$ so that $f$ is normalized by $f(0)=f_{z}(0)-1=0$. Thus, for $f=h+\bar{g} \in \mathscr{H}$, the functions $h$ and $g$ are analytic in $\mathcal{U}$ and can be expressed in the following forms:

$$
h(z)=z+\sum_{n=2}^{\infty} a_{n} z^{n}, \quad g(z)=\sum_{n=1}^{\infty} b_{n} z^{n} \quad\left(0 \leq b_{1}<1\right)
$$

and $f(z)$ is then given by

$$
f(z)=z+\sum_{n=2}^{\infty} a_{n} z^{n}+\overline{\sum_{n=1}^{\infty} b_{n} z^{n}} \quad\left(0 \leq\left|b_{1}\right|<1\right) .
$$

We note that the family $\mathscr{H}$ of orientation preserving, normalized harmonic univalent functions reduces to the well-known class $\mathcal{S}$ of normalized univalent functions if the coanalytic part of $f$ is identically zero; that is, $g \equiv 0$.

For functions $f \in \mathscr{H}$, Jahangiri et al. [2] defined Salagean operator on harmonic functions given by

$$
D^{\ell} f(z)=D^{\ell} h(z)+(-1)^{\ell} \overline{D^{\ell} g(z)},
$$

where

$$
D^{\ell} h(z)=z+\sum_{n=2}^{\infty} n^{\ell} a_{n} z^{n}, \quad D^{\ell} g(z)=\sum_{n=1}^{\infty} n^{\ell} b_{n} z^{n} .
$$

In 1975, Silverman [3] introduced a new class $\mathscr{T}$ of analytic functions of the form $f(z)=z-\sum_{n=2}^{\infty}\left|a_{n}\right| z^{n}$ and opened up a new direction of studies in the theory of univalent functions as well as in harmonic functions with negative coefficients [4]. Uralegaddi et al. [5] introduced analogous subclasses of star-like, convex functions with positive coefficients and opened up a new and interesting direction of research. In fact, they considered the functions where the coefficients are positive rather than negative real numbers. Motivated by the initial work of Uralegaddi et al. [5], many researchers (see [6-9]) introduced and studied various new subclasses of analytic functions with positive coefficients but analogues results on harmonic univalent 
functions have not been explored in the literature. Very recently, Dixit and Porwal [10] attempted to fill this gap by introducing a new subclass of harmonic univalent functions with positive coefficients.

Denote by $\mathscr{V}_{\mathscr{H}}$ the subfamily of $\mathscr{H}$ consisting of harmonic functions $f=h+\bar{g}$ of the form

$$
\begin{aligned}
& f(z)=z+\sum_{n=2}^{\infty} a_{n} z^{n}-\overline{\sum_{n=1}^{\infty} b_{n} z^{n}} \\
& \left(a_{n} \geq 0 ; \quad b_{n} \geq 0,0 \leq\left|b_{1}\right|<1\right) .
\end{aligned}
$$

Motivated by the earlier works of [11-14] on the subject of harmonic functions, in this paper an attempt has been made to study the class of functions $f \in \mathscr{V}_{\mathscr{H}}$ associated with Salagean operator on harmonic functions. Further, we obtain a sufficient coefficient condition for functions $f \in \mathscr{H}$ given by (3) and also show that this coefficient condition is necessary for functions $f \in \mathscr{V}_{\mathscr{H}}$, the class of harmonic functions with positive coefficients. Distortion results and extreme points, inclusion relations, and convolution properties and results on partial sums are discussed extensively.

For $0 \leq \lambda \leq 1,1<\gamma \leq 4 / 3$, we let $\mathscr{P}_{\mathscr{H}}^{\ell}(\lambda, \gamma)$ be a new subclass of $\mathscr{H}$, consisting of all functions of the form (3) satisfying the condition

$$
\Re\left(\frac{(1-\lambda) D^{\ell+1} f(z)+\lambda D^{\ell+2} f(z)}{(1-\lambda) D^{\ell} f(z)+\lambda D^{\ell+1} f(z)}\right)<\gamma
$$

where $D^{\ell} f(z)$ is given by (4) (see [2]). Also let $\mathscr{V}_{\mathscr{H}}^{\ell}(\lambda, \gamma)=$ $\mathscr{P}_{\mathscr{H}}^{\ell}(\lambda, \gamma) \cap \mathscr{V}_{\mathscr{H}}$.

\section{Coefficient Bounds}

In our first theorem, we obtain a sufficient coefficient condition for harmonic functions in $\mathscr{P}_{\mathscr{H}}^{\ell}(\lambda, \gamma)$.

Theorem 1. Let $f=h+\bar{g}$ be given by (3). If

$$
\begin{aligned}
& \sum_{n=2}^{\infty} n^{\ell} \frac{|1-\lambda+n \lambda|(n-\gamma)}{\gamma-1}\left|a_{n}\right| \\
& \quad+\sum_{n=1}^{\infty} n^{\ell} \frac{|1-\lambda-n \lambda|(n+\gamma)}{\gamma-1}\left|b_{n}\right| \leq 1,
\end{aligned}
$$

where $a_{1}=1$ and $1<\gamma \leq 4 / 3$, then $f \in \mathscr{P}_{\mathscr{H}}^{\ell}(\lambda, \gamma)$.

Proof. We let (8) hold for the coefficients of $f=h+\bar{g}$. It suffices to show that

$$
\left|\frac{A(z) / B(z)-1}{A(z) / B(z)-(2 \gamma-1)}\right|<1, \quad z \in \mathscr{U}
$$

where

$$
\begin{aligned}
A(z)= & (1-\lambda) D^{\ell+1} f(z)+\lambda D^{\ell+2} f(z) \\
= & z+\sum_{n=2}^{\infty} n^{\ell+1}(1-\lambda+n \lambda) a_{n} z^{n} \\
& -(-1)^{\ell} \sum_{n=1}^{\infty} n^{\ell+1}(1-\lambda-n \lambda) \overline{b_{n}} \bar{z}^{n}, \\
B(z)= & (1-\lambda) D^{\ell} f(z)+\lambda D^{\ell+1} f(z) \\
= & z+\sum_{n=2}^{\infty} n^{\ell}(1-\lambda+n \lambda) a_{n} z^{n} \\
& +(-1)^{\ell} \sum_{n=1}^{\infty} n^{\ell}(1-\lambda-n \lambda) \overline{b_{n}} \bar{z}^{n} .
\end{aligned}
$$

Substituting for $A(z)$ and $B(z)$ in (9), we get

$$
\begin{aligned}
& \mid \begin{array}{l}
A(z) / B(z)-1 \\
A(z) / B(z)-(2 \gamma-1)
\end{array} \\
& \leq\left(\sum_{n=2}^{\infty} n^{\ell}|1-\lambda+n \lambda|(n-1)\left|a_{n}\right||z|^{n-1}+(-1)^{\ell}\right. \\
& \left.\quad \times \sum_{n=1}^{\infty} n^{\ell}|1-\lambda-n \lambda|(n+1)\left|b_{n}\right||z|^{n-1}\right) \\
& \quad \times\left(2(\gamma-1)-\sum_{n=2}^{\infty} n^{\ell}|1-\lambda+n \lambda|[n-(2 \gamma-1)]\right. \\
& \quad \times\left|a_{n}\right||z|^{n-1}-(-1)^{\ell} \\
& \left.\quad \times \sum_{n=1}^{\infty} n^{\ell}|1-\lambda-n \lambda|[n+(2 \gamma-1)]\left|b_{n}\right||z|^{n-1}\right)^{-1} \\
& \leq\left(\sum_{n=2}^{\infty} n^{\ell}|1-\lambda+n \lambda|(n-1)\left|a_{n}\right|\right. \\
& \left.\quad+(-1)^{\ell} \sum_{n=1}^{\infty} n^{\ell}|1-\lambda-n \lambda|(n+1)\left|b_{n}\right|\right) \\
& \quad \times\left(2(\gamma-1)-\sum_{n=2}^{\infty} n^{\ell}|1-\lambda+n \lambda|[n-(2 \gamma-1)]\left|a_{n}\right|\right. \\
& \left.\quad-(-1)^{\ell} \sum_{n=1}^{\infty} n^{\ell}|1-\lambda-n \lambda|[n+(2 \gamma-1)]\left|b_{n}\right|\right)^{-1} .
\end{aligned}
$$

The above expression is bounded above by 1 if

$$
\begin{aligned}
& 2 \sum_{n=2}^{\infty} n^{\ell}|1-\lambda+n \lambda|(n-1)\left|a_{n}\right| \\
& \quad+2 \sum_{n=1}^{\infty} n^{\ell}|1-\lambda-n \lambda|(n+1)\left|b_{n}\right| \leq 2(\gamma-1)
\end{aligned}
$$


which is equivalent to

$$
\begin{aligned}
& \sum_{n=2}^{\infty} \frac{n^{\ell}|1-\lambda+n \lambda|(n-\gamma)}{\gamma-1}\left|a_{n}\right| \\
& \quad+\sum_{n=1}^{\infty} \frac{n^{\ell}|1-\lambda-n \lambda|(n+\gamma)}{\gamma-1}\left|b_{n}\right| \leq 1 .
\end{aligned}
$$

But (8) is true by hypothesis. Hence, $\mid(A(z) / B(z)-1) /$ $(A(z) / B(z)-(2 \gamma-1)) \mid<1, z \in \mathcal{U}$, and the theorem is proved for $A(z)$ and $B(z)$ is given by (10) and (11), respectively.

Theorem 2. For $a_{1}=1$ and $1<\gamma \leq 4 / 3, f=h+\bar{g} \epsilon$ $\mathscr{V}_{\mathscr{H}}^{\ell}(\lambda, \gamma)$ if and only if

$$
\begin{aligned}
& \sum_{n=2}^{\infty} n^{\ell} \frac{|1-\lambda+n \lambda|(n-\gamma)}{\gamma-1}\left|a_{n}\right| \\
& +\sum_{n=1}^{\infty} n^{\ell} \frac{|1-\lambda-n \lambda|(n+\gamma)}{\gamma-1}\left|b_{n}\right| \leq 1 .
\end{aligned}
$$

Proof. Since $\mathscr{V}_{\mathscr{H}}^{\ell}(\lambda, \gamma) \subset \mathscr{P}_{\mathscr{H}}^{\ell}(\lambda, \gamma)$, we only need to prove the "only if" part of the theorem. To this end, for functions $f$ of the form (6), we notice that the condition

$$
\mathfrak{R}\left(\frac{(1-\lambda) D^{\ell+1} f(z)+\lambda D^{\ell+2} f(z)}{(1-\lambda) D^{\ell} f(z)+\lambda D^{\ell+1} f(z)}\right)<\gamma .
$$

Equivalently,

$$
\begin{array}{r}
\Re\left(\left((1-\gamma) z-\sum_{n=2}^{\infty} n^{\ell}(1-\lambda+n \lambda)(n-\gamma) a_{n} z^{n}\right.\right. \\
\left.-(-1)^{2 \ell} \sum_{n=1}^{\infty} n^{\ell}(1-\lambda-n \lambda)(n+\gamma) b_{n} \bar{z}^{n}\right) \\
\times\left(z-\sum_{n=2}^{\infty} n^{\ell}(1-\lambda+n \lambda) a_{n} z^{n}\right. \\
\left.\left.+(-1)^{2 \ell} \sum_{n=1}^{\infty} n^{\ell}(1-\lambda-n \lambda) b_{n} \bar{z}^{n}\right)^{-1}\right)
\end{array}
$$

$\geq 0$.
The above required condition must hold for all values of $z$ in $\mathcal{U}$. Upon choosing the values of $z$ on the positive real axis where $0 \leq z=r<1$, we must have

$$
\begin{aligned}
& \left(1-\gamma-\sum_{n=2}^{\infty} n^{\ell}|1-\lambda+n \lambda|(n-\gamma) a_{n} r^{n-1}\right. \\
& \left.\quad-\sum_{n=1}^{\infty} n^{\ell}|1-\lambda-n \lambda|(n+\gamma) b_{n} r^{n-1}\right) \\
& \times\left(1-\sum_{n=2}^{\infty} n^{\ell}|(1-\lambda+n \lambda)| a_{n} r^{n-1}\right. \\
& \left.\quad+\sum_{n=1}^{\infty} n^{\ell}|1-\lambda-n \lambda| b_{n} r^{n-1}\right)^{-1} \\
& \geq 0 .
\end{aligned}
$$

If condition (15) does not hold, then the numerator in (18) is negative for $r$ sufficiently close to 1 . Hence, there exists $z_{0}=r_{0}$ in $(0,1)$ for which the quotient of $(18)$ is negative. This contradicts the required condition for $f \in \mathscr{V}_{\mathscr{H}}^{l}(\lambda, \gamma)$. This completes the proof of the theorem.

\section{Distortion Bounds and Extreme Points}

By routine procedure (see [10-13]), we can easily prove the following results; hence we state the following theorems without proof for functions in $\mathscr{V}_{\mathscr{H}}^{\ell}(\lambda, \gamma)$.

Theorem 3 (distortion bounds). Let $f \in \mathscr{V}_{\mathscr{H}}^{\ell}(\lambda, \gamma)$. Then for $|z|=r<1$, we have

$$
\begin{gathered}
\left(1-\left|b_{1}\right|\right) r-\frac{1}{2^{\ell}(1+\lambda)}\left(\frac{\gamma-1}{2-\gamma}-\frac{1+\gamma}{2-\gamma}\left|b_{1}\right|\right) r^{2} \leq|f(z)| \\
\quad \leq\left(1+\left|b_{1}\right|\right) r+\frac{1}{2^{\ell}(1+\lambda)}\left(\frac{\gamma-1}{2-\gamma}-\frac{1+\gamma}{(2-\gamma)}\left|b_{1}\right|\right) r^{2} .
\end{gathered}
$$

Corollary 4 (covering result). If $f(z) \in \mathscr{V}_{\mathscr{H}}^{\ell}(\lambda, \gamma)$, then

$$
\begin{aligned}
& \left\{w:|w|<\frac{2^{\ell+1}(1+\gamma)+1-\left[2^{\ell}(1+\gamma)+1\right] \gamma}{2^{\ell}(2-\gamma)(1+\lambda)}\right. \\
& \left.-\frac{2^{\ell+1}(1+\gamma)-1-\left[2^{\ell}(1+\gamma)+1\right] \gamma}{2^{\ell}(2-\gamma)(1+\lambda)}\right\} b_{1} \\
& \subset f(U) .
\end{aligned}
$$

Next we state the extreme points of closed convex hulls of $\mathscr{V}_{\mathscr{H}}^{\ell}(\lambda, \gamma)$ denoted by clco $\mathscr{V}_{\mathscr{H}}^{\ell}(\lambda, \gamma)$.

Theorem 5. A function $f(z) \in \mathscr{V}_{\mathscr{H}}^{\ell}(\lambda, \gamma)$ if and only if $f(z)=$ $\sum_{n=1}^{\infty}\left(X_{n} h_{n}(z)+Y_{n} g_{n}(z)\right)$ where $h_{1}(z)=z, h_{n}(z)=z+((\gamma-$ $\left.1) /\left(2^{\ell}|1-\lambda+n \lambda|(n-\gamma)\right)\right) z^{n},(n \geq 2)$, and $g_{n}(z)=z+((\gamma-$ 1) $\left./\left(2^{\ell}|1-\lambda-n \lambda|(n+\gamma)\right)\right) \bar{z}^{n},(n \geq 1)$ also $\sum_{n=1}^{\infty}\left(X_{n}+Y_{n}\right)=$ $1, X_{n} \geq 0$ and $Y_{n} \geq 0$. In particular, the extreme points of $\mathscr{V}_{\mathscr{H}}^{\ell}(\lambda, \gamma)$ are $\left\{h_{n}\right\}$ and $\left\{g_{n}\right\}$. 
Theorem 6. The family $\mathscr{V}_{\mathscr{H}}^{\ell}(\lambda, \gamma)$ is closed under convex combinations.

\section{Inclusion Results}

Now, we will examine the closure properties of the class $\mathscr{V}_{\mathscr{H}}^{\ell}(\lambda, \gamma)$ under the generalized Bernardi-Libera-Livingston integral operator $\mathscr{L}_{c}(f)$ which is defined by $\mathscr{L}_{c}(f)=((c+$ 1)/ $\left./ z^{c}\right) \int_{0}^{z} t^{c-1} f(t) d t, c>-1$.

Theorem 7. Let $f(z) \in \mathscr{V}_{\mathscr{H}}^{\ell}(\lambda, \gamma)$. Then $\mathscr{L}_{c}(f(z)) \in$ $\mathscr{V}_{\mathscr{H}}^{\ell}(\lambda, \gamma)$

Lemma 8 (see [15]). Let $f=h+\bar{g}$ be given by (3). If

$$
\begin{aligned}
& \sum_{n=2}^{\infty} \frac{2^{\ell}|1-\lambda+n \lambda|(n-\alpha)}{1-\alpha}\left|a_{n}\right| \\
& \quad+\sum_{n=1}^{\infty} \frac{2^{\ell}|1-\lambda-n \lambda|(n+\alpha)}{1-\alpha}\left|b_{n}\right| \leq 1,
\end{aligned}
$$

where $a_{1}=1$ and $0 \leq \alpha<1$, then $f \in \mathscr{M}_{\mathscr{H}}^{*}(\lambda, \alpha)$.

Theorem 9. Let $f=h+\bar{g} \in \mathscr{V}_{\mathscr{H}}^{\ell}(\lambda, \alpha)$ be given by (6). Then $f \in \mathscr{V}_{\mathscr{H}}^{\ell}(\lambda,(4-3 \gamma) /(3-2 \gamma))$.

Proof. Since $f \in \mathscr{V}_{\mathscr{H}}^{\ell}(\lambda, \gamma)$, then by Theorem 1 we must have

$$
\begin{aligned}
& \sum_{n=2}^{\infty} \frac{2^{\ell}|1-\lambda+n \lambda|(n-\gamma)}{\gamma-1}\left|a_{n}\right| \\
& \quad+\sum_{n=1}^{\infty} \frac{2^{\ell}|1-\lambda-n \lambda|(n+\gamma)}{\gamma-1}\left|b_{n}\right| \leq 1 .
\end{aligned}
$$

To show that $f \in \mathscr{V}_{\mathscr{H}}^{\ell}(\lambda,(4-3 \gamma) /(3-2 \gamma))$, by virtue of Lemma 8 we have to show that

$$
\begin{aligned}
& \sum_{n=2}^{\infty} \frac{2^{\ell}|1-\lambda+n \lambda|[n-((4-3 \gamma) /(3-2 \gamma))]}{1-((4-3 \gamma) /(3-2 \gamma))}\left|a_{n}\right| \\
& \quad+\sum_{n=1}^{\infty} \frac{2^{\ell}|1-\lambda-n \lambda|[n+((4-3 \gamma) /(3-2 \gamma))]}{1-((4-3 \gamma) /(3-2 \gamma))}\left|b_{n}\right| \leq 1,
\end{aligned}
$$

where $0 \leq(4-3 \gamma) /(3-2 \gamma)<1$. For this, it is sufficient to prove that

$$
\begin{aligned}
& \frac{|1-\lambda+n \lambda|(n-\gamma)}{\gamma-1} \\
& \geq \frac{|1-\lambda+n \lambda|[n-((4-3 \gamma) /(3-2 \gamma))]}{1-((4-3 \gamma) /(3-2 \gamma))}, \\
& \quad(n=2,3, \ldots),
\end{aligned}
$$

$$
\begin{aligned}
& \frac{|1-\lambda-n \lambda|(n+\gamma)}{\gamma-1} \\
& \geq \frac{|1-\lambda-n \lambda|[n+((4-3 \gamma) /(3-2 \gamma))]}{1-((4-3 \gamma) /(3-2 \gamma))}, \\
& \quad(n=1,2,3, \ldots)
\end{aligned}
$$

or equivalently $(\gamma-1)|1-\lambda+n \lambda|(n-\gamma) \geq 0(n=2,3, \ldots)$, and $(\gamma-1)|1-\lambda-n \lambda|(n+\gamma) \geq 0(n=1,2,3, \ldots)$, which is true and the theorem is proved.

Corollary 10. $\mathscr{V}_{\mathscr{H}}^{\ell}(\lambda, \gamma) \subset \mathscr{V}_{\mathscr{H}}^{\ell}(\lambda, 4 / 3) \subset \mathscr{V}_{\mathscr{H}}^{\ell}$.

\section{Convolution Properties}

For functions $f \in \mathscr{H}$ given by (3) and $F \in \mathscr{H}$ given by

$$
F(z)=H(z)+\overline{G(z)}=z+\sum_{n=2}^{\infty} A_{n} z^{n}+\overline{\sum_{n=1}^{\infty} B_{n} z^{n}},
$$

we recall the Hadamard product (or convolution) of $f$ and $F$ by

$$
(f * F)(z)=z+\sum_{n=2}^{\infty} a_{n} A_{n} z^{n}+\overline{\sum_{n=1}^{\infty} b_{n} B_{n} z^{n}} \quad(z \in \mathcal{U}) .
$$

Let $F_{j}(z) \in \mathscr{V}_{\mathscr{H}}^{\ell}(\lambda, \gamma)(j=1,2,3, \ldots, p)$ be given by

$$
F_{j}(z)=z+\sum_{n=2}^{\infty}\left|a_{n, j}\right| z^{n}+(-1)^{\ell} \overline{\sum_{n=1}^{\infty}\left|b_{n, j}\right| z^{n}},
$$

then the convolution is defined by

$$
\begin{aligned}
\left(F_{1} * \cdots * F_{p}\right)(z)= & z-\sum_{n=2}^{\infty} \prod_{j=1}^{p}\left|a_{n, j}\right| z^{n} \\
& +(-1)^{\ell} \sum_{n=1}^{\infty} \prod_{j=1}^{p}\left|b_{n, j}\right| \overline{z^{n}} .
\end{aligned}
$$

Theorem 11. Let $F_{j}(z) \in \mathscr{V}_{\mathscr{H}}^{\ell}\left(\lambda, \gamma_{j}\right)(j=1,2,3, \ldots, p)$, then $\left(F_{1} * \cdots * F_{p}\right)(z) \in \mathscr{V}_{\mathscr{H}}^{\ell}(\lambda, \beta)$, where

$$
\beta=1+\frac{\prod_{j=1}^{p}\left(\gamma_{j}-1\right)}{2^{\ell}(1+\lambda) \prod_{j=1}^{p}\left(2+\gamma_{j}\right)-\prod_{j=1}^{p}\left(\gamma_{j}-1\right)} .
$$

Proof. We use the principle of mathematical induction in our proof. Let $F_{1} \in \mathscr{V}_{\mathscr{H}}^{\ell}\left(\lambda, \gamma_{1}\right)$, and $F_{2} \in \mathscr{V}_{\mathscr{H}}^{\ell}\left(\lambda, \gamma_{2}\right)$. By using Theorem 2, we have

$$
\begin{aligned}
& \sum_{n=2}^{\infty} n^{\ell} \frac{|1-\lambda+n \lambda|\left(n-\gamma_{j}\right)}{\gamma_{j}-1}\left|a_{n, j}\right| \\
& \quad+\sum_{n=1}^{\infty} n^{\ell} \frac{|1-\lambda-n \lambda|\left(n+\gamma_{j}\right)}{\gamma_{j}-1}\left|b_{n, j}\right| \leq 1
\end{aligned}
$$


then

$$
\begin{gathered}
{\left[\sum_{n=2}^{\infty}\left(\sqrt{n^{\ell} \frac{|1-\lambda+n \lambda|\left(n-\gamma_{1}\right)}{\gamma_{1}-1}\left|a_{n, 1}\right|}\right)^{2}\right.} \\
\left.\times \sum_{n=2}^{\infty}\left(\sqrt{n^{\ell} \frac{|1-\lambda+n \lambda|\left(n-\gamma_{2}\right)}{\gamma_{2}-1}\left|a_{n, 2}\right|}\right)^{2}\right]^{1 / 2} \\
+\left[\sum_{n=1}^{\infty}\left(\sqrt{n^{\ell} \frac{|1-\lambda-n \lambda|\left(n+\gamma_{1}\right)}{\gamma_{1}-1}\left|b_{n, 1}\right|}\right)^{2}\right. \\
\left.\quad \times \sum_{n=1}^{\infty}\left(\sqrt{\frac{n^{\ell} \frac{|1-\lambda-n \lambda|\left(n+\gamma_{2}\right)}{\gamma_{2}-1}}{b_{n, 2} \mid}}\right)^{2}\right]^{1 / 2}
\end{gathered}
$$

$\leq 1$.

Thus, by applying Cauchy-Schwarz inequality, we have

$$
\begin{gathered}
\sum_{n=2}^{\infty}\left(\sqrt{n^{2 \ell} \frac{|1-\lambda+n \lambda|\left(n-\gamma_{1}\right)\left(n-\gamma_{2}\right)}{\left(\gamma_{1}-1\right)\left(\gamma_{2}-1\right)}\left|a_{n, 1} a_{n, 2}\right|}\right)^{2} \\
\leq\left[\sum_{n=2}^{\infty}\left(\sqrt{n^{\ell} \frac{|1-\lambda+n \lambda|\left(n-\gamma_{1}\right)}{\gamma_{1}-1}\left|a_{n, 1}\right|}\right)^{2}\right]^{1 / 2} \\
\quad \times\left[\sum_{n=2}^{\infty}\left(\sqrt{n^{\ell} \frac{|1-\lambda+n \lambda|\left(n-\gamma_{2}\right)}{\gamma_{2}-1}\left|a_{n, 2}\right|}\right)^{2}\right]^{1 / 2}, \\
\sum_{n=1}^{\infty}\left(\sqrt{n^{2 \ell} \frac{|1-\lambda-n \lambda|\left(n+\gamma_{1}\right)\left(n+\gamma_{2}\right)}{\left(\gamma_{1}-1\right)\left(\gamma_{2}-1\right)}\left|b_{n, 1} b_{n, 2}\right|}\right)^{2} \\
\leq\left[\sum_{n=1}^{\infty}\left(\sqrt{n^{\ell} \frac{|1-\lambda+n \lambda|\left(n+\gamma_{1}\right)}{\gamma_{1}-1}\left|b_{n, 1}\right|}\right)^{2}\right]^{1 / 2} \\
\times\left[\sum_{n=1}^{\infty}\left(\sqrt{n^{\ell} \frac{|1-\lambda+n \lambda|\left(n+\gamma_{2}\right)}{\gamma_{2}-1}\left|b_{n, 2}\right|}\right)^{2}\right]^{1 / 2} .
\end{gathered}
$$

Then, we get

$$
\begin{array}{r}
\sum_{n=2}^{\infty} \sqrt{n^{\ell} \frac{|1-\lambda+n \lambda|\left(n-\gamma_{1}\right)\left(n-\gamma_{2}\right)}{\left(\gamma_{1}-1\right)\left(\gamma_{2}-1\right)}\left|a_{n, 1} a_{n, 2}\right|} \\
+\sum_{n=1}^{\infty} \sqrt{n^{2 \ell} \frac{|1-\lambda-n \lambda|\left(n+\gamma_{1}\right)}{\gamma_{1}-1}\left|b_{n, 1} b_{n, 2}\right|}
\end{array}
$$

Therefore, if

$$
\begin{aligned}
& \sum_{n=2}^{\infty} n^{\ell} \frac{|1-\lambda+n \lambda|(n-\alpha)}{\alpha-1}\left|a_{n, 1} a_{n, 2}\right| \\
& \quad \leq \sum_{n=2}^{\infty} \sqrt{n^{2 \ell} \frac{|1-\lambda+n \lambda|\left(n-\gamma_{1}\right)\left(n-\gamma_{2}\right)}{\left(\gamma_{1}-1\right)\left(\gamma_{2}-1\right)}\left|a_{n, 1} a_{n, 2}\right|}, \\
& \sum_{n=1}^{\infty} n^{\ell} \frac{|1-\lambda-n \lambda|(n+\alpha)}{\alpha-1}\left|b_{n, 1} b_{n, 2}\right| \\
& \leq \sum_{n=1}^{\infty} \sqrt{\frac{n^{2 \ell} \frac{|1-\lambda-n \lambda|\left(n+\gamma_{1}\right)\left(n+\gamma_{2}\right)}{\left(\gamma_{1}-1\right)\left(\gamma_{2}-1\right)}\left|b_{n, 1} b_{n, 2}\right|}{\alpha}}
\end{aligned}
$$

that is, if

$$
\begin{aligned}
& \sqrt{\left|a_{n, 1} a_{n, 2}\right|} \leq \frac{\alpha-1}{n-\alpha} \sqrt{\frac{|1-\lambda+n \lambda|\left(n-\gamma_{1}\right)\left(n-\gamma_{2}\right)}{\left(\gamma_{1}-1\right)\left(\gamma_{2}-1\right)}}, \\
& \sqrt{\left|b_{n, 1} b_{n, 2}\right|} \leq \frac{\alpha-1}{n+\alpha} \sqrt{\frac{|1-\lambda-n \lambda|\left(n+\gamma_{1}\right)\left(n+\gamma_{2}\right)}{\left(\gamma_{1}-1\right)\left(\gamma_{2}-1\right)}},
\end{aligned}
$$

then $\left(F_{1} * F_{2}\right)(z) \in \mathscr{V}_{\mathscr{H}}^{\ell}(\lambda, \alpha)$. By (30) we have

$$
\begin{aligned}
& \sum_{n=2}^{\infty} \sqrt{n^{\ell} \frac{|1-\lambda+n \lambda|\left(n-\gamma_{j}\right)}{\gamma_{j}-1}\left|a_{n, j}\right|} \leq 1, \\
& \sum_{n=1}^{\infty} \sqrt{n^{\ell} \frac{|1-\lambda-n \lambda|\left(n+\gamma_{j}\right)}{\gamma_{j}-1}\left|b_{n, j}\right|} \leq 1 .
\end{aligned}
$$

Hence we get

$$
\begin{aligned}
& \sqrt{\left|a_{n, j}\right|} \leq \frac{\gamma_{j}-1}{|1-\lambda+n \lambda|\left(n-\gamma_{j}\right)} \\
& \sqrt{\left|b_{n, j}\right|} \leq \frac{\gamma_{j}-1}{|1-\lambda-n \lambda|\left(n+\gamma_{j}\right)} .
\end{aligned}
$$

Consequently, if

$$
\begin{aligned}
& \sqrt{\frac{\left(\gamma_{1}-1\right)\left(\gamma_{2}-1\right)}{n^{2 \ell}|1-\lambda+n \lambda|^{2}\left(n-\gamma_{1}\right)\left(n-\gamma_{2}\right)}} \\
& \leq \frac{\alpha-1}{n-\alpha} \sqrt{\frac{|1-\lambda+n \lambda|\left(n-\gamma_{1}\right)\left(n-\gamma_{2}\right)}{\left(\gamma_{1}-1\right)\left(\gamma_{2}-1\right)}} \\
& \sqrt{\frac{\left(\gamma_{1}-1\right)\left(\gamma_{2}-1\right)}{n^{2 \ell}|1-\lambda-n \lambda|^{2}\left(n+\gamma_{1}\right)\left(n+\gamma_{2}\right)}} \\
& \leq \frac{\alpha-1}{n+\alpha} \sqrt{\frac{|1-\lambda-n \lambda|\left(n+\gamma_{1}\right)\left(n+\gamma_{2}\right)}{\left(\gamma_{1}-1\right)\left(\gamma_{2}-1\right)}} .
\end{aligned}
$$


That is, if

$$
\begin{aligned}
& \frac{n-\alpha}{\alpha-1} \leq \frac{|1-\lambda+n \lambda|\left(n-\gamma_{1}\right)\left(n-\gamma_{2}\right)}{\left(\gamma_{1}-1\right)\left(\gamma_{2}-1\right)}, \\
& \frac{n+\alpha}{\alpha-1} \leq \frac{|1-\lambda-n \lambda|\left(n+\gamma_{1}\right)\left(n+\gamma_{2}\right)}{\left(\gamma_{1}-1\right)\left(\gamma_{2}-1\right)}
\end{aligned}
$$

then $\left(F_{1} * F_{2}\right)(z) \in \mathscr{V}_{\mathscr{H}}^{\ell}(\lambda, \alpha)$. Then we see that

$\alpha \geq 1$

$$
\begin{aligned}
& +\frac{(n-1)\left(\gamma_{1}-1\right)\left(\gamma_{2}-1\right)}{n^{\ell}|1-\lambda+n \lambda|\left(n+\gamma_{1}\right)\left(n+\gamma_{2}\right)+\left(\gamma_{1}-1\right)\left(\gamma_{2}-1\right)} \\
= & \phi(n), \\
\alpha \geq 1 & \\
& +\frac{(n+1)\left(\gamma_{1}-1\right)\left(\gamma_{2}-1\right)}{n^{\ell}|1-\lambda-n \lambda|\left(n+\gamma_{1}\right)\left(n+\gamma_{2}\right)-\left(\gamma_{1}-1\right)\left(\gamma_{2}-1\right)} \\
= & \psi(n) .
\end{aligned}
$$

Since $\phi(n)$ for $n \geq 2$ and $\psi(n)$ for $n \geq 1$ are increasing,

$$
\begin{aligned}
& \alpha \geq 1+\frac{\left(\gamma_{1}-1\right)\left(\gamma_{2}-1\right)}{2^{\ell}|1+\lambda|\left(2+\gamma_{1}\right)\left(2+\gamma_{2}\right)+\left(\gamma_{1}-1\right)\left(\gamma_{2}-1\right)} \\
& \alpha \geq 1+\frac{2\left(\gamma_{1}-1\right)\left(\gamma_{2}-1\right)}{|1-2 \lambda|\left(1+\gamma_{1}\right)\left(1+\gamma_{2}\right)-\left(\gamma_{1}-1\right)\left(\gamma_{2}-1\right)},
\end{aligned}
$$

and also

$$
\begin{aligned}
& \frac{\left(\gamma_{1}-1\right)\left(\gamma_{2}-1\right)}{2^{\ell}|1+\lambda|\left(2+\gamma_{1}\right)\left(2+\gamma_{2}\right)+\left(\gamma_{1}-1\right)\left(\gamma_{2}-1\right)} \\
& \leq \frac{2\left(\gamma_{1}-1\right)\left(\gamma_{2}-1\right)}{|1-2 \lambda|\left(1+\gamma_{1}\right)\left(1+\gamma_{2}\right)-\left(\gamma_{1}-1\right)\left(\gamma_{2}-1\right)},
\end{aligned}
$$

then $\left(F_{1} * F_{2}\right)(z) \in \mathscr{V}_{\mathscr{H}}^{\ell}(\lambda, \alpha)$ where

$$
\alpha \geq 1+\frac{\left(\gamma_{1}-1\right)\left(\gamma_{2}-1\right)}{2^{\ell}(1+\lambda)\left(2+\gamma_{1}\right)\left(2+\gamma_{2}\right)+\left(\gamma_{1}-1\right)\left(\gamma_{2}-1\right)} .
$$

Next, we suppose that $\left(F_{1} * F_{2} * \cdots * F_{p}\right)(z) \in \mathscr{V}_{\mathscr{H}}^{\ell}(\lambda, \beta)$, where

$$
\beta=1+\frac{\prod_{j=1}^{p}\left(\gamma_{j}-1\right)}{2^{\ell}(1+\lambda) \prod_{j=1}^{p}\left(2+\gamma_{j}\right)-\prod_{j=1}^{p}\left(\gamma_{j}-1\right)} .
$$

We can show that $\left(F_{1} * F_{2} * \cdots * F_{p+1}\right)(z) \in \mathscr{V}_{\mathscr{H}}^{\ell}(\lambda, \delta)$, where

$$
\delta \geq 1+\frac{(\beta-1)\left(\gamma_{p+1}-1\right)}{2^{\ell}(1+\lambda)(2+\beta)\left(2+\gamma_{p+1}\right)+(\beta-1)\left(\gamma_{p+1}-1\right)} .
$$

Since

$$
\begin{aligned}
& (\beta-1)\left(\alpha_{p+1}-1\right) \\
& =\frac{\prod_{j=1}^{p+1}\left(\gamma_{j}-1\right)}{2^{\ell}(1+\lambda) \prod_{j=1}^{p}\left(2+\gamma_{j}\right)-\prod_{j=1}^{p}\left(\gamma_{j}-1\right)}, \\
& (\beta-2)\left(\alpha_{p+1}-2\right) \\
& =\frac{\prod_{j=1}^{p+1}\left(\gamma_{j}-1\right)}{2^{\ell}(1+\lambda) \prod_{j=1}^{p}\left(2+\gamma_{j}\right)-\prod_{j=1}^{p}\left(\gamma_{j}-1\right)},
\end{aligned}
$$

we have

$$
\delta=1+\frac{\prod_{j=1}^{p+1}\left(\gamma_{j}-1\right)}{2^{\ell}(1+\lambda) \prod_{j=1}^{p+1}\left(2+\gamma_{j}\right)-\prod_{j=1}^{p+1}\left(\gamma_{j}-1\right)} .
$$

Corollary 12. Let $F_{j}(z) \in \mathscr{V}_{\mathscr{H}}^{\ell}(\lambda, \gamma)(j=1,2,3, \ldots, p)$, then $\left(F_{1} * \cdots * F_{p}\right)(z) \in \mathscr{V}_{\mathscr{H}}^{l}(\lambda, \beta)$, where

$$
\beta=1+\frac{(\gamma-1)^{p}}{2^{\ell}(1+\lambda)(2+\gamma)^{p}-(\gamma-1)^{p}} .
$$

\section{Partial Sums Results}

In 1985, Silvia [16] studied the partial sums of convex functions of order $\alpha(0 \leq \alpha<1)$. Later on, Silverman [17] and several researchers studied and generalized the results on partial sums for various classes of analytic functions only but analogues results on harmonic functions have not been explored in the literature. Very recently, Porwal [18] and Porwal and Dixit [19] filled this gap by investigating interesting results on the partial sums of star-like harmonic univalent functions. Now in this section we discussed the partial sums results for the class of harmonic functions with positive coefficients based on Salagean operator of order $\gamma(1<\gamma \leq 4 / 3)$ on lines similar to Porwal [18].

Let $\mathscr{P}_{\mathscr{H}}^{\ell}\left(A_{n} / \delta, B_{n} / \delta\right)$ denote the subclass of $\mathscr{H}$ consisting of functions $f=h+\bar{g}$ of the form (3) which satisfy the inequality

$$
\sum_{n=2}^{\infty} \frac{A_{n}}{\delta}\left|a_{n}\right|+\sum_{n=1}^{\infty} \frac{B_{n}}{\delta}\left|b_{n}\right| \leq 1
$$

where

$$
\begin{aligned}
& \frac{A_{n}}{\delta}=\frac{n^{\ell}|1-\lambda+n \lambda|(n-\gamma)}{\gamma-1}, \\
& \frac{B_{n}}{\delta}=\frac{n^{\ell}|1-\lambda-n \lambda|(n+\gamma)}{\gamma-1}
\end{aligned}
$$

and $\delta=\gamma-1$, unless otherwise stated. 
Now, we discuss the ratio of a function of the form (6) with $b_{1}=0$ being

$$
\begin{gathered}
f_{m}(z)=z+\sum_{n=2}^{m} a_{n} z^{n}+\overline{\sum_{n=1}^{\infty} b_{n} z^{n}} \\
f_{k}(z)=z+\overline{\sum_{n=2}^{\infty} a_{n} z^{n}+\overline{\sum_{n=2}^{k} b_{n} z^{n}}} \\
f_{m, k}(z)=z+\overline{\sum_{n=2}^{m} a_{n} z^{n}+}
\end{gathered}
$$

We first obtain the sharp bounds for $\mathfrak{R}\left\{f(z) / f_{m}(z)\right\}$.

Theorem 13. If $f$ of the form (6) with $b_{1}=0$ satisfies the condition (50), then

$$
\mathfrak{R}\left\{\frac{f(z)}{f_{m}(z)}\right\} \geq \frac{A_{m+1}-\delta}{A_{m+1}}, \quad(z \in \mathcal{U}),
$$

where

$$
\begin{aligned}
& A_{n} \geq \begin{cases}\delta, & \text { if } n=2,3, \ldots, m, \\
A_{m+1}, & \text { if } n=m+1, m+2, \ldots,\end{cases} \\
& B_{n} \geq \delta, \quad \text { if } n=2,3, \ldots .
\end{aligned}
$$

The result (53) is sharp with the function given by

$$
f(z)=z+\frac{\delta}{A_{m+1}} z^{m+1}
$$

Proof. Define the function $w(z)$ by

$$
\begin{aligned}
\frac{1+w(z)}{1-w(z)}= & \frac{A_{m+1}}{\delta}\left[\frac{f\left(r e^{i \theta}\right)}{f_{m}\left(r e^{i \theta}\right)}-\frac{A_{m+1}-\delta}{A_{m+1}}\right] \\
= & \left(1+\sum_{n=2}^{m} a_{n} r^{n-1} e^{i(n-1) \theta}\right. \\
& +\sum_{n=2}^{\infty} \overline{b_{n}} r^{n-1} e^{-i(n+1) \theta} \\
& \left.-\frac{A_{m+1}}{\delta}\left(\sum_{n=m+1}^{\infty} a_{n} r^{n-1} e^{i(n-1) \theta}\right)\right) \\
\times & \left(1+\sum_{n=2}^{m} a_{n} r^{n-1} e^{i(n-1) \theta}\right. \\
& \left.+\sum_{n=2}^{\infty} \overline{b_{n}} r^{n-1} e^{-i(n+1) \theta}\right)^{-1} .
\end{aligned}
$$

It suffices to show that $|w(z)| \leq 1$. Now, from (56), we can write

$$
\begin{aligned}
& w(z)=\left.\frac{A_{m+1}}{\delta}\left(\sum_{n=m+1}^{\infty} a_{n} r^{n-1} e^{i(n-1) \theta}\right)\right) \\
& \times\left(2+2\left(\sum_{n=2}^{\infty} a_{n} r^{n-1} e^{i(n-1) \theta}\right.\right. \\
&\left.+\sum_{n=2}^{\infty} \overline{b_{n}} r^{n-1} e^{-i(n+1) \theta}\right) \\
&\left.+\frac{A_{m+1}}{\delta}\left(\sum_{n=m+1}^{\infty} a_{n} r^{n-1} e^{i(n-1) \theta}\right)\right)^{-1} .
\end{aligned}
$$

Hence we obtain

$$
\begin{aligned}
& |w(z)| \\
& \quad \leq \frac{\left(A_{m+1} / \delta\right)\left(\sum_{n=m+1}^{\infty}\left|a_{n}\right|\right)}{2-2\left[\sum_{n=2}^{m}\left|a_{n}\right|+\sum_{n=2}^{\infty}\left|b_{n}\right|\right]-\left(A_{m+1} / \delta\right) \sum_{n=m+1}^{\infty}\left|a_{n}\right|} .
\end{aligned}
$$

Now $|w(z)| \leq 1$ if

$$
\sum_{n=2}^{\infty}\left|a_{n}\right|+\sum_{n=2}^{\infty}\left|b_{n}\right|+\frac{A_{m+1}}{\delta} \sum_{n=m+1}^{\infty}\left|a_{n}\right| \leq 1 .
$$

From condition (50), it is sufficient to show that

$$
\begin{gathered}
\sum_{n=2}^{\infty}\left|a_{n}\right|+\sum_{n=2}^{\infty}\left|b_{n}\right|+\frac{A_{m+1}}{\delta} \sum_{n=m+1}^{\infty}\left|a_{n}\right| \\
\leq \sum_{n=2}^{\infty} \frac{A_{n}}{\delta}\left|a_{n}\right|+\sum_{n=2}^{\infty} \frac{B_{n}}{\delta}\left|a_{n}\right|
\end{gathered}
$$

which is equivalently to

$$
\begin{gathered}
\sum_{n=2}^{m}\left(\frac{A_{n}-\delta}{\delta}\right)\left|a_{n}\right|+\sum_{n=2}^{\infty}\left(\frac{B_{n}-\delta}{\delta}\right)\left|b_{n}\right| \\
+\sum_{n=m+1}^{\infty}\left(\frac{A_{n}-A_{n+1}}{\delta}\right)\left|a_{n}\right| \geq 0 .
\end{gathered}
$$

To see that the function given by (55) gives the sharp result, we observe that for $z=r e^{i \pi / n}$

$$
\begin{aligned}
\frac{f(z)}{f_{m}(z)} & =1+\frac{\delta}{A_{m+1}} z^{m} \longrightarrow 1-\frac{\delta}{A_{m+1}} \\
& =\frac{A_{m+1}-\delta}{A_{m+1}} \quad \text { when } r \longrightarrow 1^{-} .
\end{aligned}
$$

We next determine bounds for $\mathfrak{R}\left\{f_{m}(z) / f(z)\right\}$.

Theorem 14. If $f$ of the form (6) with $b_{1}=0$ satisfies condition (50), then

$$
\mathfrak{R}\left\{\frac{f_{m}(z)}{f(z)}\right\} \geq \frac{A_{m+1}}{A_{m+1}+\delta}, \quad(z \in \mathcal{U}),
$$


where

$$
\begin{aligned}
& A_{n} \geq \begin{cases}\delta, & \text { if } n=2,3, \ldots, m, \\
A_{m+1}, & \text { if } n=m+1, m+2, \ldots,\end{cases} \\
& B_{n} \geq \delta, \quad \text { if } n=2,3, \ldots .
\end{aligned}
$$

The result (63) is sharp with the function given by

$$
f(z)=z+\frac{\delta}{A_{m+1}} z^{m+1} .
$$

Proof. Define the function $w(z)$ by

$$
\begin{aligned}
\frac{1+w(z)}{1-w(z)}= & \frac{A_{m+1}+\delta}{\delta}\left[\frac{f_{m}\left(r e^{i \theta}\right)}{f\left(r e^{i \theta}\right)}-\frac{A_{m+1}}{A_{m+1}+\delta}\right] \\
= & \left(1+\sum_{n=2}^{m} a_{n} r^{n-1} e^{i(n-1) \theta}\right. \\
& +\sum_{n=2}^{\infty} \overline{b_{n}} r^{n-1} e^{-i(n+1) \theta} \\
& \left.-\frac{A_{m+1}}{\delta}\left(\sum_{n=m+1}^{\infty} a_{n} r^{n-1} e^{i(n-1) \theta}\right)\right) \\
\times & \left(1+\sum_{n=2}^{m} a_{n} r^{n-1} e^{i(n-1) \theta}\right. \\
& \left.+\sum_{n=2}^{\infty} \overline{b_{n}} r^{n-1} e^{-i(n+1) \theta}\right)^{-1} .
\end{aligned}
$$

Hence we obtain

$$
\begin{aligned}
|w(z)| \leq & \left(\frac{A_{m+1}+\delta}{\delta}\left(\sum_{n=m+1}^{\infty}\left|a_{n}\right|\right)\right) \\
& \times\left(2-2\left[\sum_{n=2}^{m}\left|a_{n}\right|+\sum_{n=2}^{\infty}\left|b_{n}\right|\right]\right. \\
& \left.\quad-\left(\left(A_{m+1}-\delta\right) / \delta\right) \sum_{n=m+1}^{\infty}\left|a_{n}\right|\right)^{-1}
\end{aligned}
$$

$$
\leq 1
$$

The last inequality is equivalent to

$$
\sum_{n=2}^{\ell}\left|a_{n}\right|+\sum_{n=2}^{\infty}\left|b_{n}\right|+\frac{A_{m+1}}{\delta} \sum_{n=m+1}^{\infty}\left|a_{n}\right| \leq 1 .
$$

Making use of (50) and the condition (64), we obtain (61). Finally, equality holds in (63) for the extremal function $f(z)$ given by (65).

We next turns to ratios for $\mathfrak{R}\left\{f^{\prime}(z) / f_{m}^{\prime}(z)\right\}$ and $\Re\left\{f_{m}^{\prime}(z) / f^{\prime}(z)\right\}$.
Theorem 15. If $f$ of the form (6) with $b_{1}=0$ satisfies condition (50), then

$$
\mathfrak{R}\left\{\frac{f^{\prime}(z)}{f_{m}^{\prime}(z)}\right\} \geq \frac{A_{m+1}-(m+1) \delta}{A_{m+1}}, \quad(z \in \mathcal{U}),
$$

where

$$
\begin{aligned}
& A_{n} \geq \begin{cases}\delta, & \text { if } n=2,3, \ldots, m, \\
A_{m+1}, & \text { if } n=m+1, m+2, \ldots,\end{cases} \\
& B_{n} \geq \delta, \quad \text { if } n=2,3, \ldots .
\end{aligned}
$$

The result (69) is sharp with the function given by $f(z)=z+$ $\left(\delta / A_{m+1}\right) z^{m+1}$.

Proof. Define the function $w(z)$ by

$$
\begin{aligned}
\frac{1+w(z)}{1-w(z)}= & \frac{A_{m+1}}{(m+1) \delta}\left[\frac{f^{\prime}(z)}{f_{m}^{\prime}(z)}-\frac{A_{m+1}-(m+1) \delta}{A_{m+1}}\right] \\
= & \left(1+\sum_{n=2}^{m} n a_{n} r^{n-1} e^{i(n-1) \theta}\right. \\
& +\sum_{n=2}^{\infty} n \overline{b_{n}} r^{n-1} e^{-i(n+1) \theta} \\
& \left.-\frac{A_{m+1}}{(m+1) \delta}\left(\sum_{n=m+1}^{\infty} n a_{n} r^{n-1} e^{i(n-1) \theta}\right)\right) \\
\times & \left(1+\sum_{n=2}^{m} n a_{n} r^{n-1} e^{i(n-1) \theta}\right. \\
& \left.-\sum_{n=2}^{\infty} n \overline{b_{n}} r^{n-1} e^{-i(n+1) \theta}\right)^{-1} .
\end{aligned}
$$

The result (69) follows by using the techniques as used in Theorem 13.

Proceeding exactly as in the proof of Theorem 14, we can prove the following theorem.

Theorem 16. If $f$ of the form (6) with $b_{1}=0$ satisfies the condition (50), then

$$
\mathfrak{R}\left\{\frac{f_{m}^{\prime}(z)}{f^{\prime}(z)}\right\} \geq \frac{A_{m+1}}{A_{m+1}+(m+1) \delta}, \quad(z \in \mathcal{U}) .
$$

The result is sharp with the function given by $f(z)=z+$ $\left(\delta / A_{m+1}\right) z^{m+1}$.

We next determine bounds for $\mathfrak{R}\left\{f(z) / f_{k}(z)\right\}$ and $\Re\left\{f_{k}(z) / f(z)\right\}$.

Theorem 17. If $f$ of the form (6) with $b_{1}=0$ satisfies condition (50), then

$$
\mathfrak{R}\left\{\frac{f(z)}{f_{k}(z)}\right\} \geq \frac{B_{k+1}-\delta}{B_{k+1}}, \quad(z \in \mathcal{U}),
$$


where

$$
\begin{aligned}
& B_{n} \geq \begin{cases}\delta, & \text { if } n=2,3, \ldots, k, \\
B_{k+1}, & \text { if } n=k+1, k+2, \ldots,\end{cases} \\
& A_{n} \geq \delta, \quad \text { if } n=2,3, \ldots .
\end{aligned}
$$

The result (73) is sharp with the function given by $f(z)=z+$ $\left(\delta / B_{k+1}\right) \bar{z}^{k+1}$.

Theorem 18. If $f$ of the form (6) with $b_{1}=0$ satisfies condition (50), then

$$
\mathfrak{R}\left\{\frac{f_{k}(z)}{f(z)}\right\} \geq \frac{B_{k+1}}{B_{k+1}+\delta}, \quad(z \in \mathcal{U}),
$$

where

$$
\begin{aligned}
& B_{n} \geq \begin{cases}\delta, & \text { if } n=2,3, \ldots, k, \\
B_{k+1}, & \text { if } n=k+1, k+2, \ldots,\end{cases} \\
& A_{n} \geq \delta, \quad \text { if } n=2,3, \ldots .
\end{aligned}
$$

The result (75) is sharp with the function given by $f(z)=z+$ $\left(\delta / B_{k+1}\right) \bar{z}^{k+1}$.

Proof. Define the function $w(z)$ by

$$
\begin{aligned}
\frac{1+w(z)}{1-w(z)}= & \frac{B_{k+1}+\delta}{\delta}\left[\frac{f_{k}\left(r e^{i \theta}\right)}{f\left(r e^{i \theta}\right)}-\frac{B_{k+1}}{B_{k+1}+\delta}\right] \\
= & \left(1+\sum_{n=2}^{\infty} a_{n} r^{n-1} e^{i(n-1) \theta}+\sum_{n=2}^{k} \overline{b_{n}} r^{n-1} e^{-i(n+1) \theta}\right. \\
& \left.-\frac{B_{k+1}}{B_{k+1}+\delta} \sum_{n=2}^{k+1} \overline{b_{n}} r^{n-1} e^{-i(n-1) \theta}\right) \\
& \times\left(1+\sum_{n=2}^{\infty} a_{n} r^{n-1} e^{i(n-1) \theta}\right. \\
& \left.+\sum_{n=2}^{k} \overline{b_{n}} r^{n-1} e^{-i(n+1) \theta}\right)^{-1} .
\end{aligned}
$$

We omit the details of proof, because it runs parallel to that from Theorem 14.

Theorem 19. If $f$ of the form (6) with $b_{1}=0$ satisfies condition (50), then

$$
\Re\left\{\frac{f(z)}{f_{m, k}(z)}\right\} \geq \frac{A_{m+1}-\delta}{A_{m+1}}, \quad(z \in \mathcal{U}),
$$

where

$$
\begin{aligned}
& A_{n} \geq \begin{cases}\delta, & \text { if } n=2,3, \ldots, m, m+1, \\
A_{m+1}, & \text { if } n=m+1, m+2, \ldots,\end{cases} \\
& B_{n} \geq \begin{cases}\delta, & \text { if } n=2,3, \ldots, m, \\
A_{m+1}, & \text { if } n=m+1, m+2, \ldots .\end{cases}
\end{aligned}
$$

The result (78) is sharp with the function given by $f(z)=z+$ $\left(\delta / A_{m+1}\right) z^{m+1}$.
Theorem 20. If $f$ of the form (6) with $b_{1}=0$ satisfies condition (50), then

$$
\mathfrak{R}\left\{\frac{f(z)}{f_{m, k}(z)}\right\} \geq \frac{B_{k+1}-\delta}{B_{k+1}}, \quad(z \in \mathcal{U})
$$

where

$$
\begin{gathered}
B_{n} \geq \begin{cases}\delta, & \text { if } n=2,3, \ldots, k, \\
B_{k+1}, & \text { if } n=k+1, k+2, \ldots,\end{cases} \\
A_{n} \geq \begin{cases}\delta, & \text { if } n=2,3, \ldots, k, \\
B_{k+1}, & \text { if } n=k+1, k+2, \ldots\end{cases}
\end{gathered}
$$

Theorem 21. If $f$ of the form (6) with $b_{1}=0$ satisfies condition (50), then

$$
\Re\left\{\frac{f_{m, k}(z)}{f(z)}\right\} \geq \frac{A_{m+1}}{A_{m+1}+\delta}, \quad(z \in \mathcal{U}) .
$$

Theorem 22. If $f$ of the form (6) with $b_{1}=0$ satisfies condition (50), then

$$
\Re\left\{\frac{f_{m, k}(z)}{f(z)}\right\} \geq \frac{B_{k+1}}{B_{k+1}+\delta}, \quad(z \in \mathcal{U}) .
$$

The result (83) is sharp with the function given by $f(z)=z+$ $\left(\delta / B_{k+1}\right) \bar{z}^{k+1}$.

Theorem 23. If $f$ of the form (6) with $b_{1}=0$ satisfies condition (50), then

$$
\mathfrak{R}\left\{\frac{f^{\prime}(z)}{f_{m}^{\prime}(z)}\right\} \geq \frac{A_{m+1}-(m+1) \delta}{A_{m+1}}, \quad(z \in \mathcal{U}),
$$

where

$$
\begin{aligned}
& A_{n} \geq \begin{cases}\delta, & \text { if } n=2,3, \ldots, m, \\
A_{m+1}, & \text { if } n=m+1, m+2, \ldots,\end{cases} \\
& B_{n} \geq \begin{cases}\delta, & \text { if } n=2,3, \ldots, m, \\
A_{m+1}, & \text { if } n=m+1, m+2, \ldots .\end{cases}
\end{aligned}
$$

The result (84) is sharp with the function given by $f(z)=z+$ $\left(\delta / A_{m+1}\right) z^{m+1}$.

Theorem 24. If $f$ of the form (6) with $b_{1}=0$ satisfies condition (50), then

$$
\mathfrak{R}\left\{\frac{f_{m, k}^{\prime}(z)}{f^{\prime}(z)}\right\} \geq \frac{A_{m+1}}{A_{m+1}+(m+1) \delta}, \quad(z \in \mathcal{U}) .
$$

The result (86) is sharp with the function given by $f(z)=z+$ $\left(\delta / A_{m+1}\right) z^{m+1}$.

Concluding Remarks. By choosing $\lambda=0$ (or $\lambda=1$ ) and $\ell=0$ (or $\ell=1$ ), the various results presented in this paper would provide interesting extensions and generalizations of the subclasses of harmonic star-like functions with positive coefficients of order $\gamma(1<\gamma \leq 4 / 3)$ based on Salagean operator and similarly for convex functions. The details involved in the derivations of such specializations of the results presented in this paper are fairly straight-forward and hence omitted. 


\section{Conflict of Interests}

The authors declare that there is no conflict of interests regarding the publication of this paper.

\section{Acknowledgment}

We record our sincere thanks to the referees for their valuable suggestions.

\section{References}

[1] J. Clunie and T. Sheil-Small, "Harmonic univalent functions," Annales Academiae Scientiarum Fennicae A, vol. 9, pp. 3-25, 1984.

[2] J. M. Jahangiri, G. Murugusundaramoorthy, and K. Vijaya, "Salagean-type harmonic univalent functions," Southwest Journal of Pure and Applied Mathematics, no. 2, pp. 77-82, 2002.

[3] H. Silverman, "Univalent functions with negative coefficients," Proceedings of the American Mathematical Society, vol. 51, pp. 109-116, 1975.

[4] H. Silverman, "Harmonic univalent functions with negative coefficients," Journal of Mathematical Analysis and Applications, vol. 220, no. 1, pp. 283-289, 1998.

[5] B. A. Uralegaddi, M. D. Ganigi, and S. M. Sarangi, "Univalent functions with positive coefficients," Tamkang Journal of Mathematics, vol. 25, no. 3, pp. 225-230, 1994.

[6] K. K. Dixit and V. Chandra, "On subclass of univalent functions with positive coefficients," The Aligarh Bulletin of Mathematics, vol. 27, no. 2, pp. 87-93, 2008.

[7] K. K. Dixit and A. L. Pathak, "A new class of analytic functions with positive coefficients," Indian Journal of Pure and Applied Mathematics, vol. 34, no. 2, pp. 209-218, 2003.

[8] S. Porwal and K. K. Dixit, "An application of certain convolution operator involving hypergeometric functions," Journal of Rajasthan Academy of Physical Sciences, vol. 9, no. 2, pp. 173186, 2010.

[9] S. Porwal, K. K. Dixit, V. Kumar, and P. Dixit, "On a subclass of analytic functions defined by convolution," General Mathematics, vol. 19, no. 3, pp. 57-65, 2011.

[10] K. K. Dixit and S. Porwal, "A subclass of harmonic univalent functions with positive coefficients," Tamkang Journal of Mathematics, vol. 41, no. 3, pp. 261-269, 2010.

[11] J. M. Jahangiri, "Harmonic functions starlike in the unit disk," Journal of Mathematical Analysis and Applications, vol. 235, no. 2, pp. 470-477, 1999.

[12] G. Murugusundaramoorthy and K. Vijaya, "A subclass of harmonic functions associated with wright hypergeometric functions," Advanced Studies in Contemporary Mathematics, vol. 18, no. 1, pp. 87-95, 2009.

[13] G. Murugusundaramoorthy, K. Vijaya, and R. K. Raina, "A subclass of harmonic functions with varying arguments defined by Dziok-Srivastava operator," Archivum Mathematicum, vol. 45, no. 1, pp. 37-46, 2009.

[14] S. Porwal and K. K. Dixit, "New subclasses of harmonic starlike and convex functions," Kyungpook Mathematical Journal, vol. 53, no. 3, pp. 467-478, 2013.

[15] K. Vijaya, Studies on certain subclasses of Harmonic functions [Ph.D. thesis], VIT University, Vellore, India, 2007.

[16] E. M. Silvia, "On partial sums of convex functions of order $\alpha$," Houston Journal of Mathematics, vol. 11, no. 3, pp. 397-404, 1985.
[17] H. Silverman, "Partial sums of starlike and convex functions," Journal of Mathematical Analysis and Applications, vol. 209, no. 1, pp. 221-227, 1997.

[18] S. Porwal, "Partial sums of certain harmonic univalent functions," Lobachevskii Journal of Mathematics, vol. 32, no. 4, pp. 366-375, 2011.

[19] S. Porwal and K. K. Dixit, "Partial sums of starlike harmonic univalent functions," Kyungpook Mathematical Journal, vol. 50, no. 3, pp. 433-445, 2010. 


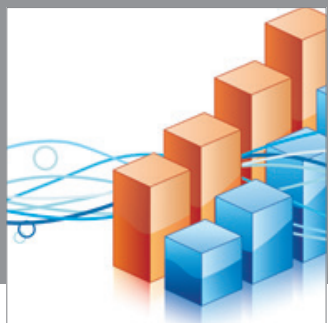

Advances in

Operations Research

mansans

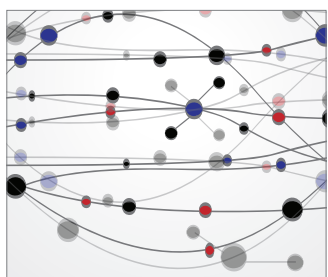

The Scientific World Journal
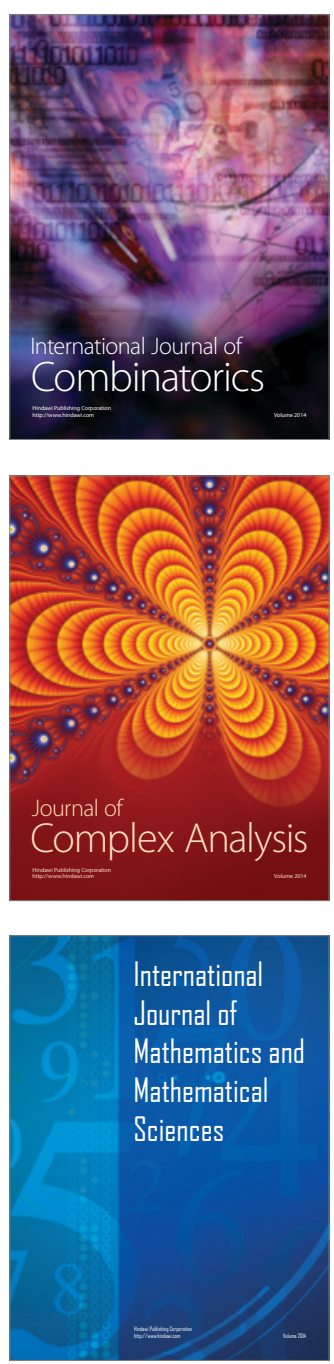
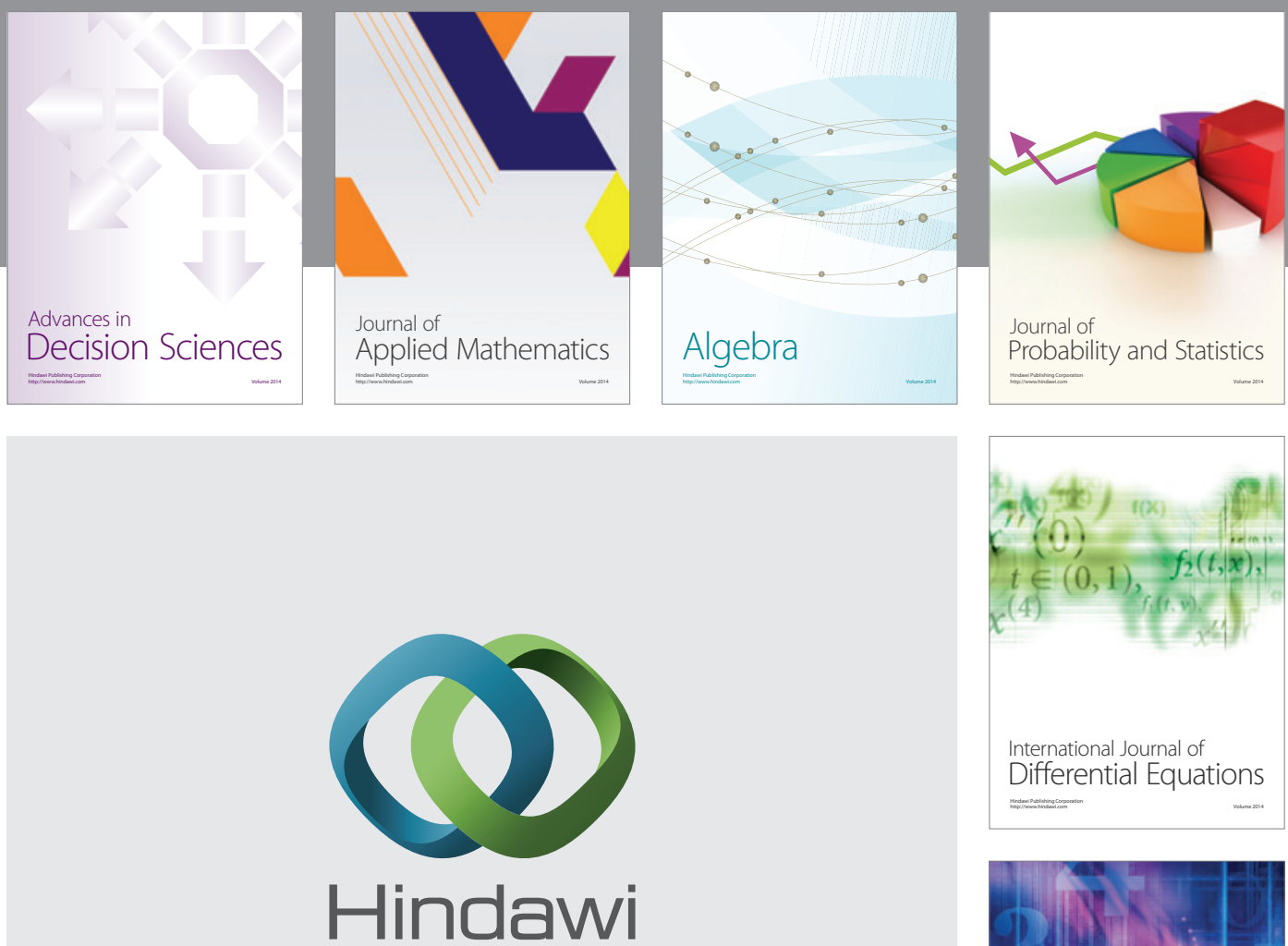

Submit your manuscripts at http://www.hindawi.com
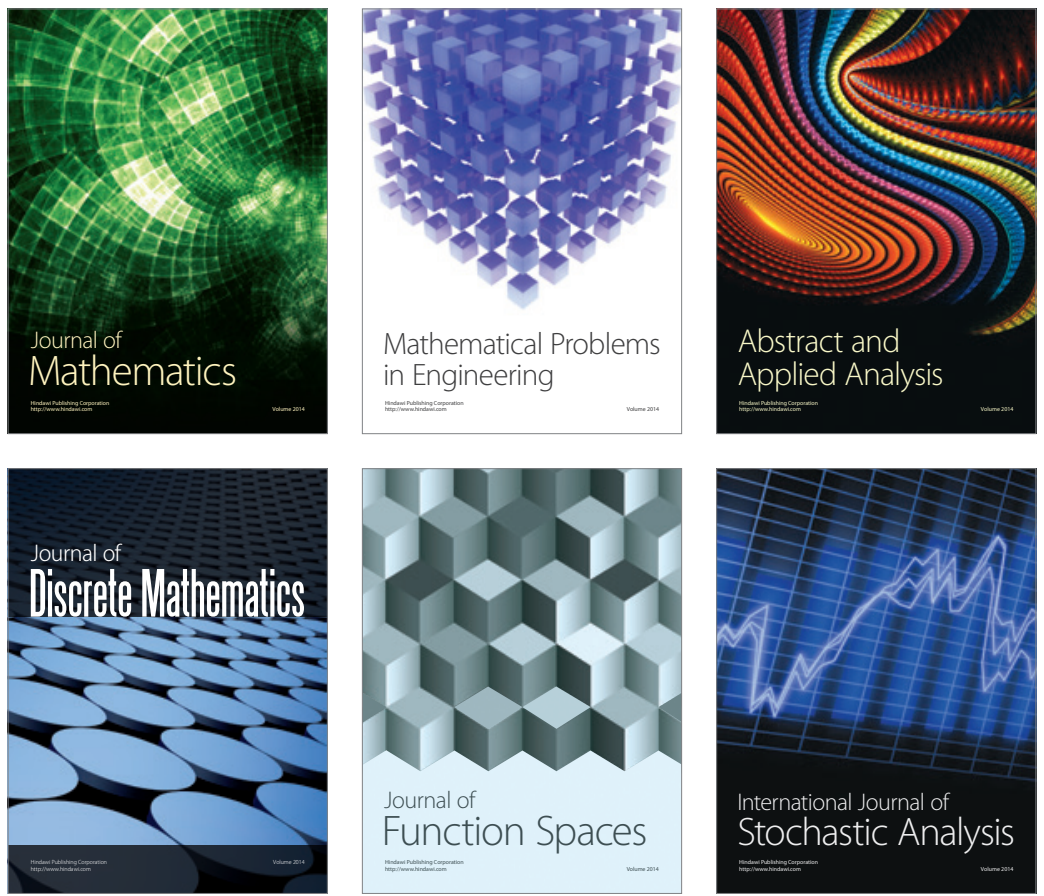

Journal of

Function Spaces

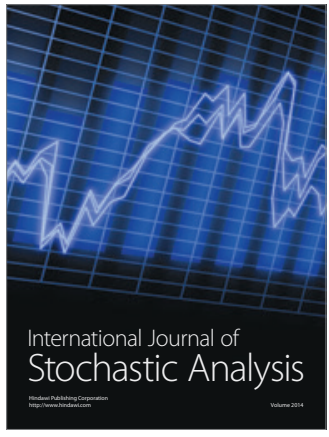

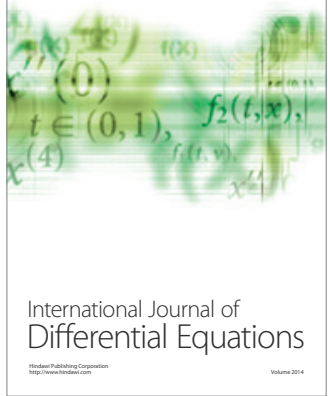
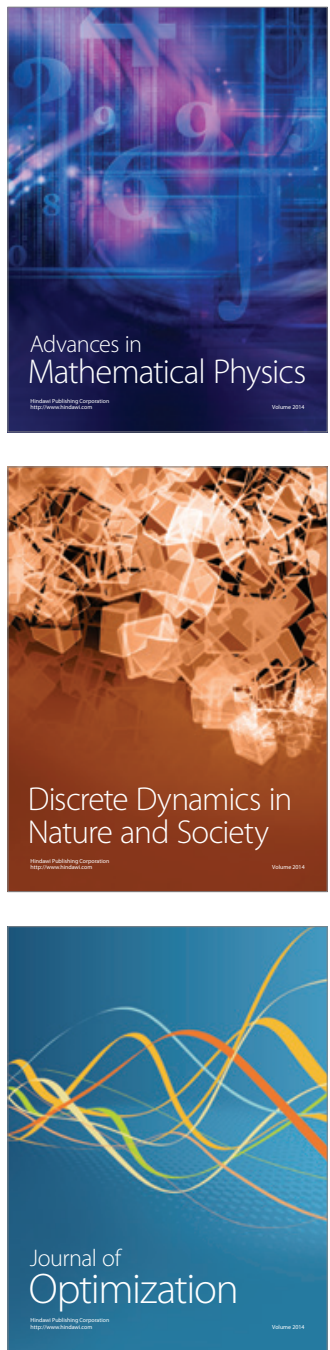